\title{
QUANTIFICAÇÃO DE GORDURA NO LEITE OVINO: ESTUDO COMPARATIVO DOS RESULTADOS OBTIDOS POR METODOLOGIA OFICIAL E POR EQUIPAMENTOS ELETRÔNICO
}

\section{Measurement of fat in the ovine milk: comparative study of the results acquired by official methodology and by electronic equipments}

\author{
Luiz Gustavo de Pellegrini ${ }^{1 *}$, Neila Silvia Pereira dos Santos Richards ${ }^{I}$
}

\begin{abstract}
RESUMO
O objetivo deste trabalho foi realizar um estudo comparativo entre o método oficial preconizado pela legislação brasileira e o equipamento eletrônico de medição fotométrica e o de espectroscopia de ultrassom na quantificação dos lipídios totais do leite ovino, a fim de verificar qual aparelho melhor expressa teor lipídico. O experimento foi desenvolvido no Departamento de Tecnologia e Ciência dos Alimentos da Universidade Federal de Santa Maria, juntamente com a Usina Escola de Laticínios e o Setor de Ovinocultura do Departamento de Zootecnia. Foram utilizadas 12 ovelhas $1 / 2$ sangue Lacaune Lait, ordenhadas individualmente da $1^{\mathrm{a}}$ a $10^{\mathrm{a}}$ semana de lactação. A ordenha foi efetuada manualmente e as análises realizadas após resfriamento das amostras. Antes de executar as análises, as amostras foram homogeneizadas e em seguida avaliadas quanto ao teor de gordura por três metodologias distintas: metodologia oficial brasileira através de butirômetro de Gerber (BG), equipamento eletrônico de medição fotométrica Milko-Tester ${ }^{\circledR}$ (MT) e equipamento de espectroscopia de ultrassom Lactoscan $90^{\circledR}$ (LS), sendo todas análises realizadas em triplicata. A reprodutibilidade do equipamento LS foi de $100 \%$ nas amostras analisadas, enquanto o equipamento MT demonstrou variabilidade na maioria das amostras analisadas, obtendo reprodutibilidade dos resultados em apenas $22,5 \%$ das amostras, sendo que nas demais amostras o mesmo obteve $50 \%$ de valores subestimados e $27,5 \%$ superestimados. Assim, os resultados deste estudo permitem
\end{abstract}

1 Universidade Federal de Santa Maria (UFSM), Av. Roraima, 1000, Cidade Universitária, Bairro Camobi, 97105-900, Santa Maria, RS, Brasil. E-mail: lgpellegrini@ibest.com.br

* Autor para correspondência.

Recebido / Received: 13/08/2015

Aprovado / Approved: 15/02/2016 
concluir que a análise de leite ovino por espectroscopia de ultrassom é eficiente para o parâmetro gordura quando comparada a metodologia oficial brasileira.

Palavras-chave: ovelha; método oficial; ultrassom; lipídios; reprodutibilidade.

\begin{abstract}
The aim of this work was to perform a comparative study between the official method recommended by Brazilian laws and the electronic equipment of photometric measurement and ultrasound spectroscopy equipment for the quantification of total lipids of the ovine milk in order to check which equipment establishes the lipids level better. The experiment took place at Technology of Food Department in Santa Maria Federal University together with School Plant of Dairy products and Ovine culture Section of Zoo Technical Department. It was used twelve sheeps half Lacaune Lait blood, milked individually from the first to the tenth week of lactation. The milking was performed manually and the analyses took place after the refrigeration of the samples. Before executing the analyses, the samples were homogenized and soon after evaluated in terms of fat amount by three distinct methodologies: official Brazilian methodology through Gerber's butyrometer (GB), electronic equipment of photometric measurement Milko-Tester ${ }^{\circledR}(\mathrm{MT})$ and ultrasound spectroscopic equipment Lactoscan $90^{\circledR}$ (LS), which all analyses were performed in triple. The reproducibility of LS equipment was $100 \%$ for the analyzed samples, while MT equipment showed variability in most of the analyzed samples obtaining reproducibility of the results in just $22,5 \%$ of the samples. For the others samples the latter equipment obtained $50 \%$ of overrated values and $27,5 \%$ underrated values. Therefore, the results of this study let us to settle that the analysis of ovine milk by ultrasound spectroscopy is efficient for the fat parameter when compared to the official Brazilian methodology.
\end{abstract}

Keywords: sheep; official method; ultrasound; lipids; reproducibility.

\section{INTRODUÇÃO}

O leite, sem outra especificação, é o produto oriundo da ordenha completa, ininterrupta, em condições de higiene, de vacas sadias, bem alimentadas e descansadas. O leite de outros animais deve denominar-se segundo a espécie de que proceda (BRASIL, 2002). Ainda, segundo Valença; Paiva (2010), o leite é um produto composto por proteínas, glicídios, lipídios, sais minerais e vitaminas, sendo a fração proteica considerada de maior importância na alimentação humana.
Dessa forma, Tronco (2003) afirma que o leite tem sido utilizado na alimentação humana por oferecer equilibrada composição de nutrientes que resulta em elevado valor biológico, sendo considerado um dos alimentos mais completos. No entanto, o conhecimento da composição do leite é essencial para a determinação de sua qualidade, pois define diversas propriedades organolépticas e industriais. Ainda, os parâmetros de qualidade são cada vez mais utilizados para detecção de falhas nas práticas de manejo, servindo como referência na valorização da matéria-prima (DÜRR, 2004). 
Segundo Monardes (1998), relata que na grande maioria dos programas de qualidade das indústrias, os teores de gordura, proteína, sólidos totais e a contagem de células somáticas que determinam a qualidade da matéria-prima. Dentre esses parâmetros podemos destacar a importância do conteúdo de proteína e gordura, pois o mesmo influencia diretamente no rendimento final de derivados lácteos.

Porém, para a análise destes parâmetros, existem vários métodos bem estabelecidos para as determinações físico-químicas, dentre os quais aqueles adotados pela legislação vigente como sendo oficiais (BRASIL, 2006), estes, exigem tempo disponível, mão-de-obra treinada e requerem reagentes caros para sua execução.

Neste sentido, Silveira et al. (2004) relatam que a utilização de equipamentos eletrônicos para análises rápidas tem sido uma alternativa para facilitar a avaliação do leite. Em vista disso, o uso dessas técnicas é de fundamental importância para os estabelecimentos beneficiadores de leite, que podem definir o destino a ser dado ao leite, subsidiar o pagamento diferenciado ao produtor com base na qualidade e assegurar que seu produto esteja adequado às normas de composição de leite exigidas pela legislação (PONSANO et al., 2007).

Entre estes equipamentos, estão os que utilizam a espectroscopia de ultrassom, onde se baseiam no princípio físico de que o movimento de qualquer onda é afetado pelo meio por onde ela está se propagando (NELLIGAN, 2003). Dessa forma, a propagação das ondas sonoras em um determinado meio fornece informações sobre ele pela análise da transmissão ou da reflexão dos sinais gerados (BHARDWAJ, 2002). Essa técnica emprega ondas sonoras de alta freqüência que imprimem forças intermoleculares aos materiais em teste. As oscilações de compressão ou descompressão das ondas ultra-sônicas causam oscilações no arranjo molecular da amostra, que responde com forças de atração ou repulsão intermoleculares (BUCKIN et al., 2003). As amplitudes de deformação nas ondas ultrasônicas empregadas na determinação são extremamente pequenas, tornando a técnica não destrutiva, o que representa uma oportunidade única na caracterização de produtos alimentícios de base líquida, amostras opacas como o leite (BUCKIN et al., 2003; DUKHIN et al., 2003; NELLIGAN, 2003).

Outro equipamento ainda utilizado é o "Milko Tester", no qual utiliza como princípio a medição fotométrica de luz dispersa pelos glóbulos de gordura presente na amostra de leite. O procedimento de medição segue o sistema convencional de diluição em ácido etilenodiamino tetra-acético (EDTA) $0,5 \%$, mistura, homogeneização e medição fotométrica.

Porém, a literatura nacional carece de informações a respeito da correlação e eficiência entre os métodos tradicionalmente e eletrônicos utilizados na determinação de componentes físico químicos do leite. Assim, o objetivo deste trabalho é realizar um estudo comparativo entre o método oficial preconizado pela legislação brasileira e, um equipamento eletrônico de medição fotométrica e de espectroscopia de ultrassom na quantificação dos lipídios totais do leite ovino a fim de verificar qual aparelho melhor expressa teor lipídico, apresentando maior eficiência e reprodutibilidade dos resultados em relação a metodologia oficial vigente.

\section{MATERIAL E MÉTODOS}

O experimento foi desenvolvido no Departamento de Tecnologia e Ciência dos Alimentos da Universidade Federal de Santa Maria, juntamente com a Usina Escola de Laticínios e o Setor de Ovinocultura do Departamento de Zootecnia. A amostragem 
ocorreu através de coletas semanais durante os meses de julho a setembro, totalizando um período de 10 semanas de lactação.

Foram utilizadas 12 ovelhas $1 / 2$ sangue Lacaune Lait, ordenhadas individualmente da $1^{\mathrm{a}}$ a $10^{\mathrm{a}}$ semana de lactação, sendo que durante o período experimental permaneceram em pasto de azevém comum (Lolium multiflorum Lam) em sistema de pastejo contínuo e água ad libitum. Ao final do dia os animais eram recolhidos em galpão coberto, onde permaneciam separados dos cordeiros por um período de 4 horas (GODFREY et al., 1997) e após esse período realizava-se a ordenha dos mesmos. Estes depois de ordenhados permaneciam no galpão onde pernoitavam e ficavam protegidos de chuva, frio e agressores.

A ordenha foi efetuada manualmente, sempre no mesmo horário e pelo mesmo ordenhador durante todo o experimento, levando em conta as boas práticas de manejo da ordenha. Após a ordenha as amostras, já identificadas, foram acondicionadas em caixa térmica com gelo, permitindo que as mesmas ficassem resfriadas até a chegada no laboratório, onde eram transferidas para refrigerador a $4{ }^{\circ} \mathrm{C}$, permanecendo até o momento das análises. Assi m, antes de executar as análises, as amostras eram expostas a temperatura ambiente do laboratório $\left(24^{\circ} \mathrm{C}\right)$ por 30 minutos, após foram homogeneizadas através de agitação mecânica por período de um minuto, e em seguida avaliadas quanto ao teor de gordura por três metodologias distintas: metodologia oficial brasileira através do butirômetro de Gerber (BG) (BRASIL, 2006), equipamento eletrônico de medição fotométrica Milko-Tester ${ }^{\circledR}$ (MT), modelo mk 3.2 (ITR), calibrado pelo método convencional de Gerber (ZAFALON, 2003) e equipamento de espectroscopia de ultrassom Lactoscan $90^{\circledR}$ (LS) (Milkotronic Ltda, Nova Zagora, Bulgária), sendo todas análises realizadas em triplicata.
O método oficial consiste em adicionar $10 \mathrm{ml}$ de ácido sulfúrico no butirômetro, colocando lentamente $11 \mathrm{ml}$ da amostra (com auxílio de uma pipeta) e, depois, somar com $1 \mathrm{ml}$ de álcool isoamílico. Na sequência, devese arrolar o butirômetro com o auxílio de luvas e agitar cuidadosamente a solução para sua completa dissolução. Levar para a centrífuga por 5 minutos, e deixar em banho-maria a $62{ }^{\circ} \mathrm{C}$ por 3 minutos com a rolha para baixo. Por fim, basta manejar a rolha para que a camada amarela (gordura) fique na escala graduada do lactobutirômetro, onde o valor obtido corresponde diretamente à porcentagem de gordura presente.

$\mathrm{Na}$ análise eletrônica por ultrassom, destinado à quantificar a porcentagem dos constituintes do leite por meio de ultrassom, o equipamento foi calibrado para a análise de leite ovino, segundo as instruções contidas no manual do equipamento. Já para o equipamento Milko-Tester as variações de gordura são percebidas pela maior ou menor opacidade das misturas produzidas. A amostra é tratada com solução de EDTA ocorrendo uma diluição da mesma, eliminando a turbidez produzida pelas micelas de caseína. A mistura de leite com a solução passa por uma fotocélula, que mede a dispersão da luz, calibrando de forma que a dispersão da solução seja proporcional à quantidade de gordura do leite (TRONCO, 2003).

Para análise estatística utilizou-se o delineamento experimental de blocos inteiramente casualizados. Os dados obtidos foram submetidos à análise de variância (ANOVA) e as médias comparadas entre si pelo teste de Dunnett, $(\mathrm{p}<0,05)$ com auxilio do programa estatístico SAS (2000).

\section{RESULTADOS E DISCUSSÃO}

Conforme exposto na Tabela 1, o equipamento de espectroscopia de ultrassom Lactoscan $^{\circledR}$ apresentou excelente reprodu- 
tibilidade dos resultados. No entanto, a metodologia pelo equipamento de medição fotométrica Milko-Tester ${ }^{\circledR}$ demonstrou variabilidade na maioria das amostras analisadas quando comparado com os valores obtidos pela metodologia oficial e de ultrassom.

Dessa forma, observa-se que a reprodutibilidade do equipamento LS foi de 100\% das amostras analisadas (Tabela 1), porém, a metodologia pelo equipamento MT possibilitou a reprodutibilidade dos resultados em apenas $20 \%$ das amostras. Nas demais amostras, o mesmo obteve $66,6 \%$, dos valores subestimados e 34,5\% superestimados (Tabela 1), demonstrando que sua reprodutibilidade e eficiência apresentam pouca confiabilidade quando utilizado para a análise do teor de gordura do leite ovino.

Os resultados obtidos pelo LS neste estudo divergem ao de Robim et al. (2012), onde comparando os resultados obtidos pela metodologia oficial e de ultrassom observaram que estes demonstram diferença significativa pela análise de variância $(\mathrm{p}>0,05)$. Já o coeficiente de correlação obtido para estes dois métodos foi elevado, apresentando um valor de $r=0,61$, o que indica uma baixa semelhança de comportamento nos resultados obtidos nos dois métodos, considerando esses dois métodos como levemente comparáveis.

Já Venturoso et al. (2007) e Ponsano et al. (2007) obtiveram resultado semelhante ao deste estudo, que, ao comparar a semelhança entre a análise do teor de gordura no leite realizada pelos métodos oficial e de ultrassom, encontraram alto coeficiente de correlação $(\mathrm{r}>0,90)$, indicando semelhança nos resultados entre esse dois métodos.

Em relação ao equipamento eletrônico Milko-Tester ${ }^{\circledR}$, este pode não ter apresentado boa reprodutibilidade devido o mesmo necessitar de calibração constante e pela variação no tamanho dos glóbulos de gordura do leite, sendo que este mede a transmitância de luz através da suspensão de glóbulos de gordura (depende do número de glóbulos), incluindo a medida de todos os componentes que existam como glóbulos insolúveis em uma suspensão de versene (EDTA 0,5\%).

Ainda, observando os coeficientes de variabilidade verifica-se que os valores encontrados para os métodos oficial (BG) e espectroscopia de ultrassom Lactoscan ${ }^{[}$ (LS) são bastante próximos e mais baixos que os da metodologia eletrônico de medição fotométrica Milko-Tester ${ }^{\circledR}$ (MT) na maioria dos casos. Isso demonstra que a consistência dos métodos BG e LS é praticamente equivalente, sendo que o método MT mostrou-se um pouco menos consistente, o que se reflete nos valores médios encontrados para o teor de gordura do leite ovino.

Entretanto, uma maior discussão sobre este assunto é difícil de ser realizada, uma vez que não existem pesquisas suficientes a respeito da comparação de metodologias oficiais e, métodos eletrônicos e de espectroscopia de ultrassom na determinação das características físico-químicas de leites. No entanto, alguns autores relatam que a análise ultrassônica em leite é bastante útil em fornecer informações sobre a composição e a estrutura dos compoponentes físico-químicos, importantes para o controle do processamento e da qualidade (BUCKIN et al., 2003; DUKHIN , et al., 2003).

Ainda é importante salientar que, em termos operacionais, a análise físico-química do leite por metodologias mais modernas como a espectroscopia de ultrassom apresenta vantagens sobre os métodos tradicionais por dispensar o preparo das amostras, utilizar volumes mínimos das amostras em estado intacto, dispensar o uso de reagentes químicos e vidrarias específicos e por fornecer o resultado em pouco tempo. 
Tabela 1 - Percentuais de gordura do leite ovino obtidos através da análise por metodologia oficial (BG), equipamento eletrônico de medição fotométrica Milko-Tester ${ }^{\circledR}(\mathrm{MT})$ e equipamento de espectroscopia de ultrassom Lactoscan $^{\circledR}$ (LS)

\begin{tabular}{|c|c|c|c|c|c|c|c|c|c|c|}
\hline \multirow{3}{*}{ Animal } & \multirow{3}{*}{ SL } & \multicolumn{9}{|c|}{ Métodos } \\
\hline & & \multicolumn{3}{|c|}{ BG } & \multicolumn{3}{|c|}{ MT } & \multicolumn{3}{|c|}{ LS } \\
\hline & & MD & $\pm \mathrm{DP}$ & $\mathrm{CV}$ & MD & $\pm \mathrm{DP}$ & $\mathrm{CV}$ & MD & $\pm \mathrm{DP}$ & $\mathrm{CV}$ \\
\hline \multirow{10}{*}{01} & 1 & 8,03 & 0,02 & 0,003 & $8,48^{*}$ & 0,06 & 0,007 & 8,01 & 0,03 & 0,003 \\
\hline & 2 & 6,18 & 0,11 & 0,017 & 6,22 & 0,01 & 0,002 & 6,21 & 0,05 & 0,008 \\
\hline & 3 & 6,09 & 0,01 & 0,002 & $6,32 *$ & 0,10 & 0,016 & 6,04 & 0,02 & 0,003 \\
\hline & 4 & 8,18 & 0,05 & 0,006 & $4,45^{*}$ & 0,01 & 0,001 & 8,19 & 0,03 & 0,004 \\
\hline & 5 & 7,52 & 0,02 & 0,002 & $6,96^{*}$ & 0,06 & 0,009 & 7,52 & 0,02 & 0,003 \\
\hline & 6 & 7,29 & 0,05 & 0,007 & $6,82 *$ & 0,06 & 0,008 & 7,22 & 0,02 & 0,003 \\
\hline & 7 & 7,88 & 0,17 & 0,021 & $7,51 *$ & 0,04 & 0,005 & 7,88 & 0,03 & 0,003 \\
\hline & 8 & 8,75 & 0,01 & 0,001 & 8,76 & 0,13 & 0,015 & 8,75 & 0,01 & 0,001 \\
\hline & 9 & 8,81 & 0,03 & 0,003 & $8,20 *$ & 0,03 & 0,004 & 8,82 & 0,03 & 0,003 \\
\hline & 10 & 8,66 & 0,05 & 0,005 & $9,00 *$ & 0,04 & 0,005 & 8,67 & 0,03 & 0,003 \\
\hline \multirow{10}{*}{02} & 1 & 6,35 & 0,50 & 0,078 & 6,11 & 0,03 & 0,005 & 6,39 & 0,06 & 0,009 \\
\hline & 2 & 7,31 & 0,03 & 0,003 & 7,26 & 0,06 & 0,008 & 7,38 & 0,02 & 0,003 \\
\hline & 3 & 7,99 & 0,02 & 0,002 & $8,52 *$ & 0,09 & 0,011 & 8,01 & 0,01 & 0,001 \\
\hline & 4 & 8,23 & 0,02 & 0,002 & $8,60^{*}$ & 0,06 & 0,007 & 8,20 & 0,01 & 0,001 \\
\hline & 5 & 7,22 & 0,02 & 0,003 & $6,57^{*}$ & 0,06 & 0,008 & 7,24 & 0,02 & 0,002 \\
\hline & 6 & 6,78 & 0,01 & 0,001 & $6,25^{*}$ & 0,09 & 0,014 & 6,83 & 0,02 & 0,002 \\
\hline & 7 & 6,98 & 0,01 & 0,001 & $6,25^{*}$ & 0,07 & 0,011 & 7,03 & 0,02 & 0,003 \\
\hline & 8 & 7,25 & 0,03 & 0,004 & $5,04^{*}$ & 0,03 & 0,005 & 7,20 & 0,02 & 0,002 \\
\hline & 9 & 7,61 & 0,16 & 0,020 & $6,59 *$ & 0,04 & 0,007 & 7,61 & 0,03 & 0,003 \\
\hline & 10 & 7,23 & 0,00 & 0,000 & $6,56^{*}$ & 0,02 & 0,003 & 7,23 & 0,03 & 0,003 \\
\hline \multirow{10}{*}{03} & 1 & 6,09 & 0,03 & 0,005 & $6,89 *$ & 0,02 & 0,002 & 6,02 & 0,04 & 0,006 \\
\hline & 2 & 6,21 & 0,01 & 0,002 & $5,98^{*}$ & 0,05 & 0,008 & 6,23 & 0,02 & 0,003 \\
\hline & 3 & 6,64 & 0,08 & 0,012 & 6,67 & 0,04 & 0,005 & 6,64 & 0,02 & 0,003 \\
\hline & 4 & 6,63 & 0,08 & 0,012 & 6,62 & 0,02 & 0,002 & 6,67 & 0,02 & 0,003 \\
\hline & 5 & 7,16 & 0,01 & 0,002 & $6,30 *$ & 0,04 & 0,007 & 7,17 & 0,04 & 0,006 \\
\hline & 6 & 6,35 & 0,30 & 0,046 & $8,05^{*}$ & 0,09 & 0,011 & 6,38 & 0,03 & 0,005 \\
\hline & 7 & 6,60 & 0,08 & 0,011 & $8,15^{*}$ & 0,09 & 0,011 & 6,60 & 0,08 & 0,012 \\
\hline & 8 & 7,34 & 0,02 & 0,003 & $6,65^{*}$ & 0,01 & 0,002 & 7,31 & 0,01 & 0,002 \\
\hline & 9 & 7,63 & 0,02 & 0,003 & $9,78 *$ & 0,21 & 0,021 & 7,58 & 0,03 & 0,003 \\
\hline & 10 & 6,71 & 0,01 & 0,001 & $7,23 *$ & 0,05 & 0,007 & 6,74 & 0,05 & 0,007 \\
\hline \multirow{6}{*}{04} & 1 & 7,56 & 0,01 & 0,001 & $7,78 *$ & 0,07 & 0,008 & 7,50 & 0,03 & 0,004 \\
\hline & 2 & 6,48 & 0,03 & 0,005 & $6,34^{*}$ & 0,01 & 0,001 & 6,48 & 0,02 & 0,002 \\
\hline & 3 & 6,87 & 0,09 & 0,013 & 6,81 & 0,08 & 0,011 & 6,90 & 0,02 & 0,003 \\
\hline & 4 & 7,60 & 0,01 & 0,001 & $7,96^{*}$ & 0,10 & 0,012 & 7,60 & 0,03 & 0,003 \\
\hline & 5 & 6,71 & 0,01 & 0,001 & $6,03 *$ & 0,03 & 0,004 & 6,74 & 0,03 & 0,004 \\
\hline & 6 & 6,37 & 0,01 & 0,001 & $5,73 *$ & 0,01 & 0,001 & 6,35 & 0,02 & 0,002 \\
\hline
\end{tabular}




\begin{tabular}{|c|c|c|c|c|c|c|c|c|c|c|}
\hline \multirow{4}{*}{04} & 7 & 7,47 & 0,37 & 0,049 & 7,96 & 0,04 & 0,005 & 7,45 & 0,04 & 0,006 \\
\hline & 8 & 7,86 & 0,13 & 0,016 & $7,25^{*}$ & 0,03 & 0,004 & 7,85 & 0,04 & 0,005 \\
\hline & 9 & 8,49 & 0,51 & 0,060 & 7,63 & 0,02 & 0,003 & 8,43 & 0,03 & 0,003 \\
\hline & 10 & 8,02 & 0,02 & 0,002 & $7,11^{*}$ & 0,02 & 0,003 & 8,02 & 0,05 & 0,006 \\
\hline \multirow{10}{*}{05} & 1 & 8,92 & 0,12 & 0,014 & $9,64^{*}$ & 0,10 & 0,010 & 8,94 & 0,04 & 0,004 \\
\hline & 2 & 10,09 & 0,06 & 0,005 & $1,07 *$ & 0,07 & 0,062 & 10,02 & 0,02 & 0,002 \\
\hline & 3 & 7,60 & 0,01 & 0,001 & 7,57 & 0,02 & 0,003 & 7,58 & 0,05 & 0,007 \\
\hline & 4 & 9,01 & 0,01 & 0,001 & $9,55^{*}$ & 0,03 & 0,003 & 9,00 & 0,03 & 0,003 \\
\hline & 5 & 7,03 & 0,03 & 0,004 & $6,28 *$ & 0,12 & 0,019 & 7,02 & 0,05 & 0,007 \\
\hline & 6 & 7,59 & 0,01 & 0,001 & 7,42 & 0,12 & 0,016 & 7,57 & 0,04 & 0,005 \\
\hline & 7 & 5,02 & 0,02 & 0,004 & $5,94^{*}$ & 0,14 & 0,024 & 5,00 & 0,03 & 0,006 \\
\hline & 8 & 7,36 & 0,01 & 0,002 & 7,49 & 1,13 & 0,151 & 7,45 & 0,02 & 0,003 \\
\hline & 9 & 7,43 & 0,04 & 0,006 & $6,47^{*}$ & 0,05 & 0,007 & 7,42 & 0,03 & 0,004 \\
\hline & 10 & 7,95 & 0,06 & 0,007 & $7,30 *$ & 0,09 & 0,012 & 7,97 & 0,02 & 0,003 \\
\hline \multirow{10}{*}{06} & 1 & 5,70 & 0,77 & 0,136 & 5,89 & 0,06 & 0,011 & 5,71 & 0,03 & 0,004 \\
\hline & 2 & 6,56 & 0,02 & 0,003 & $7,11^{*}$ & 0,05 & 0,007 & 6,62 & 0,02 & 0,003 \\
\hline & 3 & 8,73 & 0,19 & 0,021 & $9,59 *$ & 0,04 & 0,004 & 8,73 & 0,01 & 0,001 \\
\hline & 4 & 7,29 & 0,04 & 0,005 & $7,50 *$ & 0,05 & 0,007 & 7,23 & 0,03 & 0,004 \\
\hline & 5 & 6,39 & 0,00 & 0,000 & $5,91 *$ & 0,03 & 0,004 & 6,42 & 0,04 & 0,006 \\
\hline & 6 & 5,67 & 0,02 & 0,003 & $5,19^{*}$ & 0,11 & 0,021 & 5,71 & 0,02 & 0,004 \\
\hline & 7 & 7,58 & 0,02 & 0,002 & $7,35^{*}$ & 0,08 & 0,011 & 7,61 & 0,01 & 0,002 \\
\hline & 8 & 8,76 & 0,13 & 0,015 & $6,87^{*}$ & 0,09 & 0,013 & 8,84 & 0,04 & 0,004 \\
\hline & 9 & 7,17 & 0,16 & 0,022 & $8,26^{*}$ & 0,23 & 0,027 & 7,49 & 0,04 & 0,005 \\
\hline & 10 & 9,38 & 0,03 & 0,003 & $8,28 *$ & 0,10 & 0,013 & 9,43 & 0,03 & 0,003 \\
\hline \multirow{10}{*}{07} & 1 & 7,10 & 0,33 & 0,047 & $6,43^{*}$ & 0,09 & 0,013 & 7,18 & 0,04 & 0,005 \\
\hline & 2 & 6,40 & 0,02 & 0,003 & 6,34 & 0,06 & 0,009 & 6,32 & 0,02 & 0,003 \\
\hline & 3 & 6,37 & 0,03 & 0,004 & $6,67 *$ & 0,03 & 0,004 & 6,32 & 0,01 & 0,001 \\
\hline & 4 & 5,03 & 0,01 & 0,001 & $4,54^{*}$ & 0,17 & 0,037 & 5,02 & 0,02 & 0,003 \\
\hline & 5 & 5,24 & 0,01 & 0,001 & $4,91 *$ & 0,03 & 0,007 & 5,27 & 0,02 & 0,003 \\
\hline & 6 & 7,65 & 0,13 & 0,017 & $7,07^{*}$ & 0,06 & 0,008 & 7,61 & 0,02 & 0,003 \\
\hline & 7 & 7,17 & 0,03 & 0,004 & $6,81 *$ & 0,09 & 0,013 & 7,17 & 0,04 & 0,005 \\
\hline & 8 & 6,65 & 0,01 & 0,002 & 6,41 & 0,18 & 0,029 & 6,64 & 0,09 & 0,013 \\
\hline & 9 & 7,05 & 0,01 & 0,001 & $6,36^{*}$ & 0,04 & 0,006 & 7,00 & 0,02 & 0,003 \\
\hline & 10 & 7,08 & 0,02 & 0,002 & $6,43^{*}$ & 0,03 & 0,004 & 7,05 & 0,04 & 0,006 \\
\hline \multirow{10}{*}{08} & 1 & 8,69 & 0,01 & 0,001 & $9,12^{*}$ & 0,03 & 0,003 & 8,66 & 0,06 & 0,006 \\
\hline & 2 & 7,60 & 0,04 & 0,005 & $8,31 *$ & 0,07 & 0,009 & 7,61 & 0,02 & 0,002 \\
\hline & 3 & 6,30 & 0,01 & 0,001 & $6,62 *$ & 0,02 & 0,002 & 6,28 & 0,02 & 0,003 \\
\hline & 4 & 7,19 & 0,00 & 0,000 & $6,70^{*}$ & 0,10 & 0,016 & 7,23 & 0,03 & 0,003 \\
\hline & 5 & 6,10 & 0,06 & 0,009 & $5,56^{*}$ & 0,01 & 0,002 & 6,06 & 0,04 & 0,006 \\
\hline & 6 & 8,26 & 0,22 & 0,026 & $7,25^{*}$ & 0,04 & 0,005 & 8,12 & 0,05 & 0,006 \\
\hline & 7 & 7,28 & 0,00 & 0,000 & $6,94^{*}$ & 0,11 & 0,016 & 7,30 & 0,03 & 0,004 \\
\hline & 8 & 10,51 & 0,41 & 0,039 & $9,03 *$ & 0,05 & 0,006 & 10,64 & 0,06 & 0,005 \\
\hline & 9 & 5,61 & 0,02 & 0,003 & $5,14^{*}$ & 0,02 & 0,004 & 5,59 & 0,02 & 0,004 \\
\hline & 10 & 7,43 & 0,04 & 0,006 & $6,47 *$ & 0,05 & 0,007 & 7,38 & 0,02 & 0,003 \\
\hline
\end{tabular}




\begin{tabular}{|c|c|c|c|c|c|c|c|c|c|c|}
\hline \multicolumn{11}{|l|}{ continuação } \\
\hline \multirow{10}{*}{09} & 1 & & & & & 0,00 & 0,010 & 6,30 & 0,04 &, 006 \\
\hline & 2 & 5,98 & 0,09 & 0,014 & 6,05 & 0,02 & 0,003 & 5,97 & 0,03 & 0,006 \\
\hline & 3 & 6,29 & 0,06 & 0,009 & $6,66^{*}$ & 0,03 & 0,005 & 6,32 & 0,02 & 0,003 \\
\hline & 4 & 6,60 & 0,02 & 0,002 & $6,26^{*}$ & 0,07 & 0,011 & 6,59 & 0,02 & 0,003 \\
\hline & 5 & 5,52 & 0,02 & 0,003 & $5,18 *$ & 0,03 & 0,006 & 5,51 & 0,06 & 0,011 \\
\hline & 6 & 7,12 & 0,05 & 0,006 & $7,58 *$ & 0,05 & 0,006 & 7,03 & 0,05 & 0,007 \\
\hline & 7 & 6,31 & 0,00 & 0,000 & $6,16^{*}$ & 0,05 & 0,009 & 6,30 & 0,02 & 0,003 \\
\hline & 8 & 8,83 & 0,08 & 0,009 & $7,97 *$ & 0,09 & 0,011 & 8,73 & 0,04 & 0,004 \\
\hline & 9 & 6,71 & 0,01 & 0,001 & $6,59 *$ & 0,04 & 0,007 & 6,71 & 0,01 & 0,001 \\
\hline & 10 & 7,91 & 0,02 & 0,002 & $7,86^{*}$ & 0,02 & 0,002 & 7,91 & 0,02 & 0,002 \\
\hline \multirow{10}{*}{10} & 1 & 6,65 & 0,95 & 0,143 & $12,75^{*}$ & 0,06 & 0,005 & 6,77 & 0,09 & 0,013 \\
\hline & 2 & 9,34 & 0,02 & 0,002 & $9,86^{*}$ & 0,04 & 0,004 & 9,31 & 0,04 & 0,004 \\
\hline & 3 & 8,94 & 0,02 & 0,002 & $9,44^{*}$ & 0,09 & 0,009 & 8,95 & 0,02 & 0,002 \\
\hline & 4 & 3,57 & 0,01 & 0,003 & $3,28 *$ & 0,03 & 0,011 & 3,59 & 0,02 & 0,006 \\
\hline & 5 & 9,82 & 0,03 & 0,004 & $9,18^{*}$ & 0,09 & 0,010 & 9,85 & 0,09 & 0,009 \\
\hline & 6 & 8,90 & 0,09 & 0,010 & $8,45^{*}$ & 0,03 & 0,004 & 8,91 & 0,02 & 0,002 \\
\hline & 7 & 9,64 & 0,03 & 0,003 & 9,27 & 0,61 & 0,066 & 9,67 & 0,03 & 0,003 \\
\hline & 8 & 12,16 & 0,21 & 0,017 & $9,60 *$ & 0,50 & 0,052 & 11,60 & 0,48 & 0,042 \\
\hline & 9 & 9,38 & 0,03 & 0,003 & $8,28 *$ & 0,10 & 0,013 & 9,34 & 0,03 & 0,003 \\
\hline & 10 & 9,90 & 0,11 & 0,011 & $9,03 *$ & 0,05 & 0,006 & 9,94 & 0,03 & 0,003 \\
\hline \multirow{10}{*}{11} & 1 & 6,84 & 0,65 & 0,095 & 6,60 & 0,14 & 0,021 & 6,86 & 0,05 & 0,007 \\
\hline & 2 & 6,24 & 0,09 & 0,014 & 6,20 & 0,03 & 0,004 & 6,19 & 0,03 & 0,005 \\
\hline & 3 & 5,80 & 0,17 & 0,030 & 5,90 & 0,03 & 0,005 & 5,81 & 0,03 & 0,004 \\
\hline & 4 & 6,14 & 0,02 & 0,002 & $5,67 *$ & 0,01 & 0,002 & 6,11 & 0,03 & 0,004 \\
\hline & 5 & 6,23 & 0,01 & 0,002 & $5,52 *$ & 0,05 & 0,008 & 6,16 & 0,04 & 0,006 \\
\hline & 6 & 6,92 & 0,16 & 0,024 & $7,44^{*}$ & 0,04 & 0,005 & 6,97 & 0,04 & 0,005 \\
\hline & 7 & 6,25 & 0,05 & 0,008 & $5,62 *$ & 0,07 & 0,013 & 6,25 & 0,04 & 0,007 \\
\hline & 8 & 6,12 & 0,01 & 0,002 & $7,96 *$ & 0,02 & 0,003 & 6,08 & 0,02 & 0,003 \\
\hline & 9 & 5,75 & 0,02 & 0,003 & $5,37 *$ & 0,00 & 0,000 & 5,78 & 0,03 & 0,005 \\
\hline & 10 & 5,62 & 0,07 & 0,013 & $6,40^{*}$ & 0,23 & 0,036 & 5,63 & 0,01 & 0,001 \\
\hline \multirow{10}{*}{12} & 1 & 7,38 & 0,18 & 0,025 & 7,26 & 0,03 & 0,004 & 7,39 & 0,03 & 0,004 \\
\hline & 2 & 8,65 & 0,06 & 0,007 & $6,06^{*}$ & 0,06 & 0,009 & 8,68 & 0,02 & 0,002 \\
\hline & 3 & 5,83 & 0,04 & 0,006 & $6,06 *$ & 0,04 & 0,006 & 5,80 & 0,02 & 0,004 \\
\hline & 4 & 8,14 & 0,01 & 0,001 & $7,36^{*}$ & 0,07 & 0,009 & 8,09 & 0,02 & 0,002 \\
\hline & 5 & 7,28 & 0,01 & 0,001 & $7,00 *$ & 0,04 & 0,006 & 7,31 & 0,02 & 0,003 \\
\hline & 6 & 8,51 & 0,02 & 0,002 & $8,01 *$ & 0,04 & 0,005 & 8,48 & 0,04 & 0,005 \\
\hline & 7 & 6,55 & 0,06 & 0,009 & 6,40 & 0,23 & 0,036 & 6,59 & 0,06 & 0,008 \\
\hline & 8 & 5,71 & 0,02 & 0,003 & 5,75 & 0,03 & 0,004 & 5,72 & 0,02 & 0,004 \\
\hline & 9 & 6,70 & 0,01 & 0,001 & $6,09 *$ & 0,06 & 0,010 & 6,68 & 0,05 & 0,007 \\
\hline & 10 & 7,28 & 0,00 & 0,000 & $6,29 *$ & 0,02 & 0,004 & 7,30 & 0,01 & 0,001 \\
\hline
\end{tabular}

* Médias na linha diferem entre si pelo teste de Dunnett $(\mathrm{p}<0,05)$.

SL - Semanas de lactação; MD - Média; DP- Desvio padrão; CV - Coeficiente de variabilidade. 


\section{CONCLUSÕES}

Os resultados deste estudo permitem concluir que a análise de leite ovino por espectroscopia de ultrassom é eficiente para o parâmetro gordura quando comparada a metodologia oficial brasileira. Também, podese inferir que o método eletrônico de medição fotométrica não é eficiente na análise do teor de gordura nas condições que o presente estudo foi realizado.

Assim, devido reduzida quantidade de pesquisas e grande divergência de resultados torna-se necessário mais estudos a respeito das técnicas, da padronização das análises e ao funcionamento dos equipamentos utilizados, a fim de proporcionar o uso de métodos de controle de qualidade que sejam rápidos, eficientes e confiáveis.

\section{REFERÊNCIAS}

BHARDWAJ, M. C. Non contact ultrasound: the final frontier in non destructive analysis. Boalsburg: Second Wave Systems, 2002. $50 \mathrm{p}$.

BRASIL. Ministério da Agricultura, Pecuária e Abastecimento. Departamento de Inspeção de Produtos de Origem Animal. Instrução Normativa $n^{\circ} 6251$, de 2918 de setembro de 2002. Aprova os Regulamentos Técnicos de Produção, Identidade e Qualidade do Leite tipo A, do Leite tipo B, do Leite tipo C, do Leite Pasteurizado e do Leite Cru Refrigerado e o seu Regulamento Técnico da Coleta de Leite Cru Refrigerado e seu Transporte a Granel, em conformidade com os Anexos desta Instrução Normativa. Diário Oficial da República Federativa do Brasil, Brasília, 20 set. 2002. Seção 1, p. 08-13.

BRASIL. Ministério da Agricultura, Pecuária e Abastecimento. Instrução Normativa $n^{\circ}$ 68, de 12 de dezembro de 2006. Oficializa os métodos analíticos oficiais físico-químicos, para controle de leite e produtos lácteos. Oficial da República Federativa do Brasil, Brasília, 14 dez. 2006. Seção 1, p. 8.

BUCKIN, V.; O’DRISCOLL, B.; SMYTH, C. Ultrasonic spectroscopy for material analysis: recent advances. Spectroscopy Europe, v. 15, p. 20-25, 2003.

DUKHIN, A. S.; GOETZ, P. J.; TRAVERS, B. Ultrasound for characterizing liquid based food products. 1 - acoustic spectroscopy. Mount Kisco: Dispersion Technology, 2003. 26f.

DÜRR, J. W. Programa nacional de melhoria da qualidade do leite: uma oportunidade única. In: DÜRR, J. W.; CARVALHO, M. P.; SANTOS, M. V. O compromisso com a qualidade do leite no Brasil. Passo Fundo: Editora Universidade de Passo Fundo, 2004. p. $38-55$.

GODFREY, R. W. et al. Lamb growth and milk production of hair and wool sheep in a semi-arid tropical environment. Small Ruminant Research, v. 24, n. 2, p. 77-83, 1997.

MONARDES, H. Programa de pagamento de leite por qualidade em Quebec, Canadá. In: SIMPÓSIO INTERNACIONAL SOBRE QUALIDADE DO LEITE, 1., 1998, Curitiba. Anais... Curitiba: Universidade Federal do Paraná, 1998. p. 40-43.

NELLIGAN, T. J. An introduction to ultrasonic material analysis. Waltham: General Electric, 2003. 3f.

PONSANO, E. H. G et al. Correlação entre métodos tradicionais e espectroscopia de ultrassom na determinação de características físico-químicas do leite. Arquivo Brasileiro 
de Medicina Veterinária e Zootecnia, v. 59, n. 4, p. 1052-1057, 2007.

ROBIM, M. S. et al. Pesquisa de fraude no leite UHT integral comercializado no estado do Rio de Janeiro e comparação entre os métodos de análises físico-químicas oficiais e o método de ultrassom. Revista Instituto de Laticínios Cândido Tostes, n. 389, v. 67, p. 43-50, 2012.

Statistical Analysis System no ${ }^{\circ}$ SAS. SAS ${ }^{\circledR}$ user's guide: statistics. $4^{\mathrm{a}}$ ed. Version 8.1, Cary: 2000.

SILVEIRA, T. M. L. et al. Comparação entre os métodos de referência e a análise eletrônica na determinação da composição do leite bovino. Arquivo Brasileiro de Medicina Veterinária e Zootecnia, v. 56, n. 6, p. 782787, 2004.

TRONCO, V. M. Manual para inspeção da qualidade do leite. $2^{\text {a }}$. Ed. - Santa Maria: UFSM, 2003, p. 168.
VALENÇA, A. L.; PAIVA, J. E. Caracterização físico-química do leite pasteurizado fornecido pelo programa leite de Pernambuco na regiã o metropolitana do Recife. In: Jornada de Ensino, Pesquisa e Extensão, 10, 2010. Anais... Recife: UFRPE, 2010.

VENTUROSO, R. C. et al. Determinação da composição físico-química de produtos lácteos: estudo exploratório de comparação dos resultados obtidos por metodologia oficial e por ultrassom. Revista Brasileira de Ciências Farmacêuticas. v. 43, n. 4, p. 607-613, 2007.

ZAFALON, L. F. Mastite subclínica bovina por Staphylococcus aureus: qualidade e quantidade de leite secretado por quartos tratados e não tratados e relação custo/benefício do tratamento durante a lactação. 2003. 66f. (Tese em Medicina Veterinária Preventiva) - Universidade Estadual Paulista, Faculdade de Ciências Agrárias e Veterinárias, Jaboticabal, 2003. 